\title{
Enriquecimiento de la Carne de Cuy (Cavia porcellus) con Ácidos Grasos Omega-3 M ediante Dietas con Aceite de Pescado y Semillas de Sacha Inchi (Plukenetia volubilis)
}

\author{
Enrichment of Guinea Pig (Cavia porcellus) Meat with Omega-3 Fatty Acids by \\ Diets with Fish OIL and SACha Inchi (Plukenetia volubilis) SeEdS
}

Jorge Guevara V. ${ }^{1,5}$, Sergio Rojas M. ${ }^{2}$, Fernando Carcelén C. ${ }^{3}$, Luis Seminario S. ${ }^{4}$

\section{Resumen}

\begin{abstract}
El objetivo del estudio fue enriquecer la carne de cuy (Cavia porcellus) con ácidos grasos omega- 3 mediante el uso de dietas con fuentes de omega-3. Se utilizaron 48 cuyes machos de 42 días de edad y peso inicial de $615 \mathrm{~g}$. Los cuyes se asignaron al azar a cuatro tratamientos con tres repeticiones (pozas) de cuatro cuyes cada una. Los tratamientos fueron: 1) Dieta control; 2) Dieta suplementada con 1.0\% de aceite de pescado; 3 ) Dieta suplementada con $4.0 \%$ de semilla de sacha inchi (Plukenetia volubilis); y 4) Dieta suplementada con $1.0 \%$ de aceite de pescado $+4.0 \%$ de semilla de sacha inchi. La fase experimental tuvo una duración de 28 días. La carne de cuyes alimentados con la dieta con aceite de pescado presentó $1.36 \%$ de omega-3 de cadena larga $(0.63 \%$ ácido eicosapentaenoico [EPA] $+0.73 \%$ ácido docosahexaenoico [DHA]) y aquella con dieta con aceite de pescado más sacha inchi alcanzó $0.99 \%$ de omega-3 $(0.44 \%$ EPA $+0.55 \%$ DHA). Las carnes de cuyes alimentados con la dieta control o con semillas de sacha inchi no presentaron omega-3 de cadena larga EPA/DHA, pero si omega-3 de cadena corta $\alpha$-linolénico (ALA). Asimismo, la carne de cuyes alimentados con la dieta con sacha inchi exhibió el más alto contenido de ácidos grasos poliinsaturados $(51.35 \%)$ y el menor de ácidos grasos monoinsaturados $(21.97 \%)$ y de ácidos grasos saturados (25.49\%). Se concluye que la dieta con aceite de pescado produjo en la carne de cuy una retención de ácidos grasos omega-3 EPA y DHA, mientras que la dieta con semillas de sacha inchi solo hubo retención de omega-3 ALA.
\end{abstract}

Palabras clave: cuy, ácidos grasos, omega 3, DHA, EPA

1 Escuela Académica Profesional de Ingeniería Agroindustrial, Universidad Nacional Mayor de San Marcos, Lima, Perú

${ }^{2}$ Departamento de Nutrición, Facultad de Zootecnia, Universidad Nacional Agraria La Molina, Lima, Perú

${ }^{3}$ Facultad de Medicina Veterinaria, Universidad Nacional Mayor de San Marcos, Lima, Perú

${ }^{4}$ Universidad de Concepción, Chillán, Chile

${ }^{5}$ E-mail: guevaravet@hotmail.com

Recibido: 30 de setiembre de 2014

Aceptado para publicación: 3 de setiembre de 2015 
The aim of this study was to enrich guinea pig (Cavia porcellus) meat with omega3 polyunsaturated fatty acids by diets with omega-3 sources. A total of 48 male guinea pigs, 42 day old and $615 \mathrm{~g}$ body weight were used. The guinea pigs were randomly assigned to four treatments with three replicates (pens) with four individuals each. The dietary treatments were: 1) Control diet; 2) $1.0 \%$ fish oil supplemented diet; 3) $4.0 \%$ sacha inchi seeds (Plukenetia volubilis) supplemented diet; and 4) $1.0 \%$ fish oil $+4.0 \%$ sacha inchi seeds supplemented diet. The experiment lasted 28 days. The meat of guinea pig supplemented with fish oil contained $1.36 \%$ large chain omega- 3 fatty acids $(0.63 \%$ eicosapentaenoic acid [EPA] $+0.73 \%$ docosahexaenoic acid [DHA]) and those supplemented with the fish oil + sacha inchi had $0.99 \%$ large chain omega-3 fatty acids $(0.44 \% \mathrm{EPA}+0.55 \% \mathrm{DHA})$. Guinea pigs on the control diet and those supplemented with sacha inchi seeds contained no large chain omega-3 fatty acids (EPA/DHA) but short chain omega-3 fatty acid $\alpha$-linolenic acid (ALA). Furthermore, meat of guinea pig fed on the sacha inchi diet showed the highest level of polyunsaturated fatty acids $(51.35 \%)$, the lowest content of monounsaturated fatty acids (21.97\%) and the lowest concentration of saturated fatty acids $(25.49 \%)$. It is concluded that the diet with fish oil produced retention of omega-3 EPA and DHA in guinea pig meat, while the diet with sacha inchi seeds only retained omega-3 ALA.

Key words: guinea pig; fatty acids; omega 3; DHA; EPA

\section{INTRODUCCIÓN}

Los ácidos grasos de cadena larga omega-3 (omega-3) son favorables para el desarrollo del cerebro y muy eficaces en la prevención de problemas cardiovasculares; esto último por sus efectos antiaterogénico y antitrombótico (De Caterina, 2011; Bonafinia et al., 2015; Smesny et al., 2015). Además, participan en la reducción del desarrollo de ciertas formas de cáncer (Messler et al., 2015).

Los animales superiores y el hombre dependen del suministro en la dieta de omega-3, debido a que no sintetizan dobles enlaces en la posición 3 de los ácidos grasos. Por esto, se recomienda un consumo diario mínimo de $1.5 \mathrm{~g}$ de ácido eicosapentaenoico (EPA o $\mathrm{C}_{20: 5}$, omega-3) o de ácido docosahexaenoico (DHA o $\mathrm{C}_{22: 6}$ omega-3) (Saldanha et al., 2009). Asimismo, se ha establecido que un consumo diario mínimo de $2.22 \mathrm{~g} / \mathrm{d}$ de áci- do a-linolénico (ALA o $\mathrm{C}_{18: 3}$ omega-3) es lo adecuado en personas adultas (Kris-Etherton et al., 2009).

En los Estados Unidos de Norteamérica y en Europa se utiliza la linaza como fuente de omega-3 de cadena corta (Kaul et al., 2008) y en el Perú se emplea el sacha inchi (Plukenetia volubilis Linneo) (Maurer et al., 2012), donde ambos productos presentan un alto contenido en ácidos grasos esenciales, como ALA (omega-3) y linoleico (AL: omega-6), poco abundantes en la naturaleza y muy importantes en la prevención y cuidado de la salud. Asimismo, estos productos pueden ser utilizados en la dieta de los animales para enriquecer su carne con estos nutrientes (Vicente et al., 2015).

El objetivo de la presente investigación fue producir carne de cuy con mayor nivel de ácidos grasos omega-3, mediante la suplementación de dietas con aceite de pescado y semillas de sacha inchi. 


\section{Materiales y Métodos}

El presente trabajo se llevó a cabo en la Sala Experimental de Cuyes del Programa de Investigación en Carnes, de la Facultad de Zootecnia, Universidad Nacional Agraria La Molina (UNALM), Lima.

Se utilizaron 12 pozas construidas de material noble revestidas con cemento. Se emplearon 48 cuyes de genotipo Cieneguilla, con una edad promedio de 42 días, peso inicial de $615 \mathrm{~g}$ e identificados con aretes de aluminio. Los cuyes provinieron de la granja de cuyes de Cieneguilla de la UNALM.

Se utilizó un diseño completamente al azar con cuatro tratamientos y tres repeticiones cada uno, donde una repetición fue representada por un grupo de cuatro cuyes alojados en una poza.

Los cuyes fueron pesados individualmente y distribuidos al azar, formando grupos homogéneos de cuatro animales por poza. Se les administró concentrado y como forraje verde el rastrojo de brócoli. Para la medición de peso vivo de los animales, alimento balanceado, forraje y carcasa, se utilizó una balanza de $5 \mathrm{~kg}$ de capacidad con $2 \mathrm{~g}$ de aproximación.

Para el suministro de concentrado, en las primeras dos semanas se utilizaron comederos de arcilla enlozada de forma rectangular, con capacidad de $250 \mathrm{~g}$; luego fueron cambiadas por tolvas plásticas pequeñas de $3 \mathrm{~kg}$ de capacidad hasta el final de la fase experimental. Como bebederos se utilizaron pocillos de porcelana con capacidad de 200 $\mathrm{ml}$ de agua por poza.

Las dietas, en forma de harina, fueron preparadas cada cuatro días y se ofrecieron a los animales diariamente ad libitum entre las 09:30 y las 10:30 horas. Las dietas fueron colocadas en bolsas plásticas convenientemente identificadas por tratamiento y almacenadas en refrigeración. Se suministró agua fresca y limpia a todos los animales diariamente entre las 10:00 y las 14:00 horas; luego se retiraban los bebederos hasta el siguiente día para evitar exceso de humedad en las camas.

Todos los cuyes consumieron una ración de crecimiento $(2730 \mathrm{Kcal}$ ED/kg y $18 \%$ de proteína cruda) durante las primeras cuatro semanas, en tanto que en las cuatro últimas recibieron una de las cuatro dietas experimentales (etapa de acabado): T0: Dieta control (sin aceite de pescado ni semilla de sacha inchi); T1: Dieta suplementada con $1.0 \%$ de aceite de pescado; T2: Dieta suplementada con $4.0 \%$ de semilla de sacha inchi; y T3: Dieta suplementada con $1.0 \%$ de aceite de pescado y $4.0 \%$ de semilla de sacha inchi. Las cuatro dietas de acabado contenían $2760 \mathrm{Kcal}$ de ED/kg y $16 \%$ de proteína cruda.

Tres cuyes por tratamiento fueron sacrificados al final del experimento, previo ayuno, para el análisis de cromatografía de los ácidos grasos. Las carcasas se colocaron en bolsas plásticas de polietileno, y remitidas al laboratorio del Instituto Tecnológico Pesquero (ITP) del Ministerio de la Producción, Lima, para la determinación del perfil de omega-3 y del contenido y calidad de la grasa de las carcasas.

\section{Resultados y Discusión}

\section{Ácidos Grasos Omega-3: EPA/DHA}

En el Cuadro 1 se presentan los contenidos de omega-3, expresados como porcentajes de grasa y en $\mathrm{mg} / 100 \mathrm{~g}$ de carne. Se observa que la carne de los cuyes alimentados con la dieta de aceite de pescado contiene $1.36 \%$ de omega-3 $(0.73 \%$ de DHA y $0.63 \%$ de EPA) y la carne de los cuyes alimentados con la dieta conteniendo aceite de pescado y semilla de sacha inchi registró un valor de $0.99 \%$ de omega-3 (0.55\% de DHA y $0.44 \%$ de EPA). Esto, en términos absolu- 
Cuadro 1. Perfil de ácidos grasos omega-3 en la carne de cuyes que fueron sometidos a dietas enriquecidas con omega-3

\begin{tabular}{lccccccccc}
\hline \multirow{2}{*}{ Ácidos grasos insaturados $^{1}$} & \multicolumn{3}{c}{$\mathrm{T}^{2}$} & \multicolumn{2}{c}{$\mathrm{T} 1$} & \multicolumn{2}{c}{$\mathrm{T} 2$} & \multicolumn{2}{c}{$\mathrm{T} 3$} \\
\cline { 2 - 9 } & $\%$ & $\mathrm{mg} / 100 \mathrm{~g}$ & $\%$ & $\mathrm{mg} / 100 \mathrm{~g}$ & $\%$ & $\mathrm{mg} / 100 \mathrm{~g}$ & $\%$ & $\mathrm{mg} / 100 \mathrm{~g}$ \\
\hline Eicosapentaenoico (EPA) & $\mathrm{ND}^{3}$ & $\mathrm{ND}$ & 0.63 & 130 & $\mathrm{ND}$ & $\mathrm{ND}$ & 0.44 & 67 \\
Docosahexaenoico (DHA) & $\mathrm{ND}$ & $\mathrm{ND}$ & 0.73 & 150 & $\mathrm{ND}$ & $\mathrm{ND}$ & 0.55 & 83 \\
Total Omega 3 (EPA + DHA) & $\mathrm{ND}$ & $\mathrm{ND}$ & 1.36 & 280 & $\mathrm{ND}$ & $\mathrm{ND}$ & 0.99 & 150 \\
a Linolénico (omega-3) & 5.45 & 1032 & 5.82 & 1199 & 12.92 & 1705 & 10.20 & 1546 \\
Total EPA + DHA + ALA & 5.45 & 1032 & 7.18 & 1479 & 12.92 & 1705 & 11.19 & 1696 \\
Linoleico (Omega-6) & 36.41 & 6892 & 32.72 & 6741 & 37.33 & 4925 & 35.43 & 5369 \\
Omega-6: Omega-3 & $\mathrm{ND}$ & $7: 1$ & $\mathrm{ND}$ & $4: 1$ & $\mathrm{ND}$ & $3: 1$ & $\mathrm{ND}$ & $3: 1$ \\
Oleico (Omega-9) & 23.43 & 4435 & 20.9 & 4306 & 19.74 & 2604 & 20.66 & 3131 \\
\hline
\end{tabular}

${ }^{1}$ Los valores corresponden a una muestra compuesta de tres carcasas de cuyes por tratamiento

${ }^{2} \mathrm{T0}=$ Dieta control; T1: Dieta suplementada con $1.0 \%$ de aceite de pescado; T2: Dieta suplementada con $4.0 \%$ de semilla de sacha inchi; y T3: Dieta suplementada con $1.0 \%$ de aceite de pescado y $4.0 \%$ de semilla de sacha inchi

${ }^{3}$ No detectado

tos correspondieron a valores de 280 y 150 mg de (EPA+DHA)/100 g de carne, respectivamente. Contrariamente, en las carnes de los cuyes que recibieron la dieta control y la dieta con semilla de sacha inchi solo se detectaron valores de ALA.

Morgan y Noble (1992) encontraron en jamones de cerdos valores superiores de omega-3 (1.6\% total, $0.8 \%$ EPA y $0.8 \%$ DHA). Estos animales fueron alimentados con dietas que contenían $0.95 \%$ de aceite de pescado y $2.5 \%$ de harina de pescado, de allí que la mayor cantidad de harina de pescado haya influenciado estos resultados.

El contenido de omega-3 en huevos es aún superior. Baltazar (2000) encontró $410 \mathrm{mg}$ omega-3 (330 mg EPA y 80 mg DHA)/100 g de huevos de codornices que consumieron una dieta con $2.65 \%$ de aceite crudo de pescado y $7.8 \%$ de harina de pescado especial. Asimismo, Barboza (1995) obtuvo $458 \mathrm{mg}$ omega-3 (82 mg EPA y $376 \mathrm{mg}$ DHA)/100 g en huevo de gallina con una dieta de $2 \%$ de aceite crudo de pescado y $10 \%$ de harina de pescado, y $418 \mathrm{mg}$ omega-3 (62 mg EPA y $356 \mathrm{mg}$ DHA) /100 g con una dieta conteniendo $2 \%$ de aceite acidulado de pescado y $10 \%$ de harina de pescado.

Los mayores valores de EPA y DHA/ $100 \mathrm{~g}$ de huevos de codornices y gallinas pudieron deberse al uso de mayores niveles de aceite de pescado (de 2.0 a 3.0\%) y de harina de pescado (de 7.8 y $10.0 \%$ ) en las dietas de dichos experimentos. Estos resultados sugieren que mayores niveles de aceite y de harina de pescado en las dietas de cuyes podrían elevar las concentraciones de EPA y DHA en su carne.

\section{Ácidos Grasos Omega-3: Ácido a Linolénico (ALA)}

Los valores de omega 3 ALA (Cuadro 1) en la carne de cuyes que recibieron la dieta con semilla de sacha inchi (12.92\% ALA) y la dieta con semilla de sacha inchi más aceite de pescado (10.20\% ALA) fueron mayores en 58 y $46.5 \%$ a los de la dieta Control $(5.45 \%$ ALA). La explicación reside en el contenido 
Cuadro 2. Ácidos grasos presentes en la carne de cuyes, según la dieta suministrada

\begin{tabular}{lcccccc}
\hline \multirow{2}{*}{ Dietas } & \multicolumn{2}{c}{ Saturados } & \multicolumn{2}{c}{ Monoinsaturados } & \multicolumn{2}{c}{ Poliins aturados } \\
\cline { 2 - 7 } & $\%$ & $\mathrm{mg} / 100 \mathrm{~g}$ & $\%$ & $\mathrm{mg} / 100 \mathrm{~g}$ & $\%$ & $\mathrm{mg} / 100 \mathrm{~g}$ \\
\hline $\begin{array}{l}\text { Control } \\
\begin{array}{l}\text { Con 1\% de aceite de } \\
\text { pescado }\end{array}\end{array}$ & 32.42 & 5763 & 26.09 & 4939 & 42.88 & 8116 \\
$\begin{array}{l}\text { Con 4\% de semilla de sacha } \\
\text { inchi }\end{array}$ & 25.49 & 3363 & 21.97 & 2899 & 51.35 & 6774 \\
$\begin{array}{l}\text { Con 1\% de aceite de } \\
\text { pescado y 4\% de semilla de } \\
\text { sacha inchi }\end{array}$ & 26.81 & 4063 & 24.26 & 3676 & 47.90 & 7258 \\
\hline${ }^{1}$ Los valores corresponden a una muestra compuesta de tres carcasas de cuyes por tratamiento
\end{tabular}

porcentual de ALA (45.2\%) en la dieta con semilla de sacha inchi, en comparación con el 5.8 y $6.4 \%$ de ALA con las dietas de aceite de pescado y la dieta control, respectivamente.

El total de omega-3 también puede calcularse considerando el ALA. Generalmente, el EPA y el DHA provienen de una fuente marina (por ejemplo, el aceite de pescado) y el ALA proviene del forraje que consumen los animales. En situaciones normales del metabolismo celular, por ejemplo cuando no existe un exceso de omega-6, el ALA puede ser precursor del EPA y DHA (Schuchardta et al., 2014).

\section{Ácidos Grasos Saturados e Insaturados}

En el Cuadro 2 se presentan los valores porcentuales de ácidos grasos saturados, monoinsaturados y poliinsaturados de la carne de cuy, según el tipo de dieta. En general, el grupo de cuyes que recibió la dieta con semilla de sacha inchi presentó en la carne el mayor nivel de ácidos grasos poliinsaturados (51.35\%), el menor nivel de ácidos monoinsaturados $(21.97 \%)$ y el menor contenido de ácidos grasos saturados $(25.49 \%)$. Por otro lado, el grupo de cuyes que recibió la dieta con aceite de pescado tuvo el nivel más bajo de ácidos grasos poliinsaturados (41.30\%) y el más alto de ácidos monoinsaturados (24.9\%) y de ácidos grasos saturados (32.42\%). En parte, estos resultados se deberían a que mientras el aceite de sacha inchi tiene $82.0 \%$ de ácidos grasos poliinsaturados, el aceite de pescado tiene $34.3 \%$.

Murphy et al. (1997) obtuvieron similares resultados, reportando una mayor proporción de ácidos grasos poliinsaturados sobre los ácidos grasos saturados y estos a su vez sobre los monoinsaturados.

\section{Conclusiones}

- La dieta con aceite de pescado produjo en la carne de cuy una retención de ácidos grasos omega-3 EPA y DHA, mientras que la dieta con semillas de sacha inchi solo hubo retención de ácidos grasos omega-3 ALA.

- La carne de cuyes suplementados con sacha inchi presentó el mayor porcentaje de ácidos grasos poliinsaturados y la menor cantidad de ácidos grasos saturados. 


\section{Literatura Citada}

1. Baltazar C. 2000. Efecto de dos niveles de ácidos grasos omega 3 de la dieta sobre la composición del huevo y el comportamiento productivo en codornices. Tesis de Ingeniero Zootecnista. Lima: Univ Nacional Agraria La Molina. 79 p.

2. Barboza V. 1995. Inclusión de aceite de pescado acidulado estabilizado. Tesis de Ingeniero Zootecnista. Lima: Univ Nacional Agraria La Molina. $86 \mathrm{p}$.

3. Bonafinia S, Antoniazzi F, Maffeis $C$, Minuz P, Fava C. 2015. Beneficial effects of $\omega-3$ PUFA in children on cardiovascular riskfactors during childhood and adolescence. Prostag Oth Lipid Mediath 120: 72-79. doi: 10.1016/ j.prostaglandins.2015.03.006

4. De Caterina R. 2011. n-3 fatty acids in cardiovascular disease. N Engl J Med 364: 2439-2450. doi: 10.1056/ NEJMra1008153

5. Kaul N, Kreml R, Austria JA, Richard $M N$, Edel AL, Dibrov E, Hirono $S$, et al. 2008. A comparison of fish oil, flaxseed oil and hempseed oil supplementation on selected parameters of cardiovascular health in healthy volunteers. J Am Coll Nutr 27: 51-58.

6. Kris-Etherton PM, Grieger JA, Etherton TD. 2009. Dietary reference intakes for DHA and EPA. Prostag Leukotr Ess 81: 99-104. doi: 10.1016/ j.plefa.2009.05.011

7. Maurer NE, Hatta-Sakoda B, Pascual-Chaqman G, .RodriguezSaona LE. 2012. Characterization and authenti-cation of a novel vegetable source of omega-3 fatty acids, sacha inchi (Plukenetia volubilis L.) oil. Food Chem 134: 1173-1180. doi: 10.1016/ j.foodchem.2012.02.143
8. Morgan C, Noble R. 1992. Manipulation of the fatty acid composition of pig meat lipids by dietary means. J Sci Food Agric 58: 357-368. doi: 10.1002/ jsfa.2740580310

9. Murphy MG, Wright V, Ackman RG, Horackova M. 1997. Diets enriched in menhaden fish oil, seal oil, or shark liver oil have distinct effects on the lipid and fatty-acid composition of guinea pig heart. Moll Cell Biochem 177: 257-269.

10. Saldanha LG, Salem Jr N, Brenna JT. 2009. Workshop on DHA as a required nutrient: overview. Prostag Leukotr Ess 81: 233-236. doi: 10.1016/j.plefa. 2009.07.001

11. Schuchardt JP, Schneider I, Willenberg I, Yang J, Hammock BD, Hann A, Schebb NH. 2014. Increase of EPA-derived hydroxy, epoxy and dihydroxy fatty acid levels in human plasma after a single dose of long-chain omega-3 PUFA. Prostag Oth Lipid Mediath 111: 23-31. doi: 10.1016/ j.prostaglan-dins.2014.03.001

12. Smesny S, Milleit B, Schaefer MR, Hipler UC, Milleit $C$, Wiegand $C$, Hesse J, et al. 2015. Effects of omega-3 PUFA on the vitamin $\mathrm{E}$ and glutathione antioxidant defense system in individuals at ultra-high risk of psychosis. Prostag Leukotr Ess 101: 1521. doi: 10.1016/j.plefa.2015.07.001

13. Vara-Messler M, Buccellati C, Pustina L, Folco G, Rovati GE, Hoxha M. 2015. A potential role of PUFAs and COXIBs in cancer chemoprevention. Prostag Oth Lipid Meditah 120: 97-102. doi: 10.1016/ j.prostaglandins.2015. 04.003.

14. Vicente J, de Carvalho MG, GarciaRojas EE. 2015. Fatty acids profile of sacha inchi oil and blends by $1 \mathrm{H}$ NMR and GC-FID. Food Chem 181: 215-221. doi: 10.1016/j.foodchem.2015.02.092. 